\title{
Effect of procedural volume on outcome of coronary artery bypass graft surgery in Japan: Implication toward public reporting and minimal volume standards
}

\author{
Hiroaki Miyata, MD, Noboru Motomura, PhD, MD, Yuichi Ueda, PhD, MD, Hikaru Matsuda, PhD, MD, and
}

Shinichi Takamoto, PhD, MD

Earn CME credits at http:// cme.ctsnetjournals.org

See related editorial on page 1202.
From the Departments of Healthcare Quality Assessment and Cardiothoracic Surgery, Graduate School of Medicine, University of Tokyo, Tokyo, Japan, the Department of Cardiothoracic Surgery, Graduate School of Medicine, Nagoya University, Nagoya, Japan, and Hyogo Medical College, Hyogo, Japan.

Received for publication May 22, 2007; revisions received Oct 21, 2007; accepted for publication Oct 26, 2007.

Address for reprints: Hiroaki Miyata, 7-3-1 Hongo, Bunkyo-ku, Tokyo 113-8655, Japan (E-mail: hiroaki.miyata@gmail.com).

J Thorac Cardiovasc Surg 2008;135:130612

\section{$0022-5223 / \$ 34.00$}

Copyright $\odot 2008$ by The American Association for Thoracic Surgery

doi:10.1016/j.jtcvs.2007.10.079
Background: Since the Japanese government updated the medical practice laws, each hospital has to submit procedural volume from April 2007 and may sometime in the future have to submit some outcome indicators. It is very important to examine whether procedural volume is accurate and appropriate.

Methods: We analyzed 4581 procedures from 36 centers between 2003 and 2005 by clinical database. The effect of hospital volume on each outcome was tested by a hierarchical mixed-effects logistic regression model, covering clinical risk factors, procedural year, clinical processes, and hospital volume/surgeon volume as a fixed effect and random intercepts for sites.

Results: Logistic regression model revealed a significant association between hospital bypass graft volume and 30-day mortality $(P<.05)$ and operative mortality $(P<.01)$. Surgeon procedural volume, however, did not have a significant effect on those outcomes. The effect of hospital procedural volume was associated with better outcomes in most patient subgroups: age younger than 65 years $(P<.05)$, age 65 years and older $(P<.01)$, low risk $(P=.58)$, and high risk $(P<.01)$.

Conclusion: In Japan, high-volume compared with low-volume providers had better outcomes. As for public reporting in Japan, hospital-based evaluation might be more credible than surgeon-based evaluation. Although minimal volume standards might be effective to improve quality to some extent, volume has limitations as a marker of quality because of its wide range of variance.

$\mathrm{S}$ ince the Japanese government updated the medical practice laws in June 2006, each local government has had the power to force medical centers to submit and bring forward "certain information" that is useful for patients choosing a hospital from April 2007 (http://www.mhlw.go.jp/topics/bukyoku/soumu/houritu/dl/ 164-4a.pdf).

As of January 2007, "certain information" includes procedural volume but few outcome indicators, such as operative mortality rate or morbidity rate. However, there is the possibility that "certain information" could include surgeon-specific outcome indicators similar to public reporting in New York State. ${ }^{1,2}$ In Japan, it is very important to examine whether procedural volume is information that is appropriate to reveal and whether it is accurate.

Measuring and understanding the association between surgical volume and outcome in the delivery of health services has been the focus of much research since the 1980s in the United States. ${ }^{3,4}$ Recently, two systematic reviews suggested that high volume is associated with better outcomes, but the degree of this association varies greatly. ${ }^{5,6}$ As the complications included in these findings are partly due to methodologic shortcomings in many studies, it is very important to conduct a rigorous examination of volume-outcome association.

In Japan, whereas 9 studies suggest that a significant relationship between volume and outcomes does exist, ${ }^{7-15} 4$ studies suggest that no such relationship exists. ${ }^{16-19}$ 


\section{Abbreviations and Acronyms \\ $\mathrm{CABG}=$ coronary artery bypass grafting \\ JACVSD = Japanese Adult Cardiovascular Surgery Database \\ JATS = Japanese Association for Thoracic Surgery \\ STS $=$ The Society of Thoracic Surgeons}

Moreover, none of those Japanese studies examines the relationships of hospital and physician volume, appropriateness of patient selection, or risk adjustment by risk model with good calibration (Hosmer-Lemeshow test positive) and discrimination ( $\mathrm{C}$-index $>0.75)$. No association between hospitals' coronary artery bypass graft (CABG) surgery volume and outcome has been reported in Japan.

We undertook a contemporary examination of the association between hospital CABG procedural volume and outcome using clinical data available from the Japanese Adult Cardiovascular Surgery Database (JACVSD). The data collection form is almost identical with that of The Society of Thoracic Surgeons (STS) National Cardiac Database. We examined whether hospital volume and surgeon volume were associated with each outcome category (30-day mortality, 3-day operative mortality). We also examined how the association between hospital CABG volume and operative mortality varied as a function of patient age and predicted surgical risk.

As JACVSD participating hospitals did not cover all centers in Japan, we also examined the database of the Japanese Association for Thoracic Surgery (JATS). Although it was hard to adjust patient preoperative risk because of its aggregate data form, the JATS survey covered nearly all centers in Japan. We considered the nationwide trend and the potential health policy implications of using hospital volume in the context of health policy.

\section{Methods}

Survey 1 (JATS Database)

Study population. Since 1986, the JATS has conducted annual surveys of thoracic surgery. JATS sent out survey questionnaires to all institutions conducting cardiovascular surgery in Japan, and the response rates of the survey were very high $(95.9 \%$ in 2001 , $97.4 \%$ in $2002,94.3 \%$ in 2003 , and $90.3 \%$ in 2004). ${ }^{20-23}$ The definitions of terms are based on the published guidelines of the STS and The American Association for Thoracic Surgery. ${ }^{24} \mathrm{We}$ examined isolated $\mathrm{CABG}$ surgery procedures, excluding those combined with valve or other major surgical interventions, performed between January 1, 2001, and December 31, 2004. We included 540 centers that reported at least one CABG procedure during 2001 through 2004. Because of the very high response rate of the JATS survey, 540 centers represent nearly all institutions in Japan doing coronary procedures.

Statistical analysis. The primary yardstick of outcome used by JATS was 30-day mortality, defined as death within 30 days of operation, regardless of the patient's geographic location. Although this criterion includes death within 30 days of operation even when the patient had been discharged from the hospital during those 30 days, patients who died in the hospital at greater than 30 days were ungraspable in the JATS survey. Hospital-isolated CABG annual case volume was averaged over a 4-year period (2001-2004) to increase its stability. Annual hospital procedural volume was divided into quarters $(15 \leq, 16-30,31-50$, and $>50)$. The break points were chosen to form 4 fairly equal-sized hospital samples, and similar volume differences were maintained among the groups. We simply showed average mortality rate (and 95\% confidence interval) per hospital (Table 1).

\section{Survey 2 (JACVSD)}

Study population. The JACVSD was established in 2000 to report surgical outcomes after cardiothoracic procedures in detail. The database currently captures clinical information from 151 hospitals ( $28.5 \%$ of all centers performing CABG surgery). The data collection form has 255 variables in total, and these variables are almost identical to the STS National Database (available online at http:// wts.org). The definitions of JACVSD variables (available online at http://www.jacvsd.umin.jp) are the same as those of the STS National Database. JACVSD constructed the software for the Webbased data collection system, and through this system each data manager in the participating hospitals submits data by computer. Although participation in the JACVSD is voluntary, data completeness is high, with overall preoperative risk factors used in risk models missing in fewer than $2 \%$. The accuracy of the submitted data is checked through data auditing in monthly visits to each hospital by administrative office members. After checking the data using

TABLE 1. Hospital outcomes and characteristics in JATS database (2001-2004)

\begin{tabular}{lccccc}
\hline & \multicolumn{4}{c}{ Hospital CABG volume (procedures per year) } & Overall \\
\cline { 2 - 5 } & $\mathbf{1 5}$ & $\mathbf{1 6 - 3 0}$ & $\mathbf{3 1 - 5 0}$ & $\leq \mathbf{5 1}$ & 82,611 \\
\hline No. of patients & 4,140 & 13,589 & 19,337 & 45,545 & 540 \\
No. of hospitals & 133 & 153 & 123 & 131 & 11,556 \\
Status emergency & 295 & 1,727 & 3,141 & 6,393 & 1,585 \\
Thirty-day mortality & 124 & 349 & 412 & 700 & 14.0 \\
Emergency rate, \% & 7.1 & 12.7 & 16.2 & 14.0 & 1.92 \\
Total mortality rate, \% & 3.00 & 2.57 & 2.13 & 1.54 & $1.61(1.80-1.43)$ \\
Average mortality rate, \% (95\% CI) & $3.79(2.11-5.48)$ & $2.60(2.17-3.01)$ & $2.17(1.85-2.49)$ & \\
\hline
\end{tabular}

$\mathrm{Cl}$, Confidence interval. 
TABLE 2. Patient characteristics of JACVSD (2003-2005)

\begin{tabular}{|c|c|c|c|c|}
\hline & \multicolumn{3}{|c|}{ Hospital CABG volume (procedures per year) } & \multirow[b]{2}{*}{ All } \\
\hline & $16-30$ & $31-50$ & $\geq \mathbf{5 1}$ & \\
\hline No. of patients & 894 & 1645 & 2042 & 4581 \\
\hline No. of hospitals & 13 & 14 & 9 & 36 \\
\hline Age, median (IOR) & $69.0(63-75)$ & $69.0(62-74)$ & $69.0(61-74)$ & $69.0(62-74)$ \\
\hline Preop creatine, median (IQR) & $0.9(0.7-1.2)$ & $0.9(0.8-1.1)$ & $0.9(0.8-1.1)$ & $0.9(0.8-1.1)$ \\
\hline Men, $\%$ & 78.2 & 76.7 & 76.9 & 77.1 \\
\hline Chronic lung disease, $\%$ & 7.4 & 5.0 & 5.5 & 5.7 \\
\hline Cerebrovascular disease, $\%$ & 10.0 & 11.8 & 12.3 & 11.7 \\
\hline Hypertension, \% & 77.4 & 70.2 & 70.5 & 71.7 \\
\hline Diabetes, \% & 48.2 & 47.8 & 46.2 & 47.2 \\
\hline Left main artery disease, $\%$ & 38.6 & 31.2 & 36.1 & 34.9 \\
\hline Three-vessel disease, $\%$ & 69.7 & 68.8 & 66.3 & 67.8 \\
\hline NYHA class IV, $\%$ & 12.9 & 12.3 & 8.4 & 10.7 \\
\hline Congestive heart failure, $\%$ & 16.3 & 17.9 & 11.6 & 14.8 \\
\hline Shock, \% & 5.7 & 6.1 & 3.6 & 4.9 \\
\hline Reoperation, \% & 3.5 & 3.9 & 2.4 & 3.1 \\
\hline Emergency/salvage, \% & 7.8 & 7.4 & 7.3 & 7.5 \\
\hline Preoperative risk, \% & 2.4 & 2.0 & 1.7 & 2.0 \\
\hline Thirty-day mortality & 2.68 & 1.95 & 1.47 & 1.88 \\
\hline Operative mortality & 4.14 & 2.86 & 1.62 & 2.55 \\
\hline
\end{tabular}

IQR, Interquartile range; NHYA, New York Heart Association. Preoperative risk was calculated on the basis on JACVSD 30-day mortality risk model. Number of patients is for 3-year periods (2003-2005).

the clinical records and operative notes, the audit members suggest that the hospital data manager complete and improve the data entry. The registration rate of JACVSD data has further been confirmed in independent comparisons of hospital CABG surgery volume submitted to the JACVSD against those reported to the JATS database. We excluded 11 centers that entered data for fewer than of the total number of cases compared with those in the JATS database. Inclusion of cases from these excluded centers strengthened the effect of procedural volume on outcome.

We examined isolated CABG surgery procedures, excluding those combined with valve surgery or other major surgical interventions, performed between January 1, 2003, and December 31, 2005. Fifty centers were members of JACVSD as of January 1, 2003. After excluding 11 centers for the aforementioned reason and excluding 3 centers because of extremely low $\mathrm{CABG}$ volume (reported $<15$ CABG procedures per year), we ultimately included the data from 36 centers (Table 2 ).

\section{TABLE 3. The effect of volume index on each outcome}

\begin{tabular}{lcc}
\hline \multicolumn{1}{c}{ Volume index (procedures per year) } & $\begin{array}{c}\text { Thirty-day } \\
\text { mortality }\end{array}$ & $\begin{array}{c}\text { Operative } \\
\text { mortality }\end{array}$ \\
\hline Hospital adult cardiac surgery volume & $<.05$ & $<.01$ \\
Hospital CABG-related surgery volume & $<.05$ & $<.01$ \\
Hospital CABG isolated volume & $<.05$ & $<.01$ \\
Surgeon adult cardiac surgery volume & $\mathrm{NA}$ & $\mathrm{NA}$ \\
Surgeon CABG-related surgery volume & $\mathrm{NA}$ & $\mathrm{NA}$ \\
Surgeon CABG isolated volume & $\mathrm{NA}$ & $\mathrm{NA}$ \\
\hline
\end{tabular}

$C A B G$, Coronary artery bypass grafting; $N A$, not significant.
Statistical analysis. The primary outcome measure of JACVSD analysis was 30-day operative mortality, defined as in-hospital or 30 -day mortality, whichever was longer. ${ }^{25}$ The annual case volumes of hospitals and surgeons were averaged over a 3-year period (2003-2005) with a view to maintaining stability. Although volume was considered to be a continuous variable in this analysis, the patient and hospital characteristics and unadjusted outcomes were categorized by annual hospital procedural volume for display purposes. The break points were identical with those of JATS analysis (16-30, 31-50, and >50).

The effect of hospital volume on unadjusted outcomes was tested by a hierarchical mixed-effects logistic regression model. We examined the 6 types of volume index in this study: hospital adult cardiac surgery volume (CABG, valve, thoracic aorta, and other procedures), hospital CABG-related surgery volume (CABG plus valve or other procedures), CABG-only surgery volume per hospital, adult cardiac surgery volume per surgeon, CABG-related surgery volume per surgeon, and CABG-only surgery volume per surgeon (Table 3). In Japan, isolated CABG surgery accounts for $48 \%$ of all adult cardiac surgery, while $29 \%$ was valvular heart disease and $19 \%$ was thoracic aortic aneurysm. ${ }^{22}$ These analyses included previously identified clinical risk factors, ${ }^{1}$ procedure year, clinical process (off-pump CABG surgery, autologous blood transfusion), hospital procedural volume, surgeon volume as a fixed effect, and random intercepts for sites. ${ }^{26}$ The C-indexes for this model in the study

\footnotetext{
${ }^{1}$ Motomura N, Miyata H, Takamoto S, Tsukihara H, Okada M, Japan Cardiovascular Surgery Database Organization. Japan Adult Cardiovascular Surgery Database: 30-day Operative Mortality and Morbidity Risk Models of CABG-only Surgery. Unpublished data.
} 
TABLE 4. Risk-adjusted mortality, by hospital and surgeon volume $(n=4581)$

\begin{tabular}{|c|c|c|c|c|c|c|c|c|}
\hline \multirow[b]{3}{*}{ Surgeon CABG volume (procedures per year) } & \multicolumn{6}{|c|}{ Hospital CABG volume (procedures per year) } & & \\
\hline & \multicolumn{2}{|c|}{$16-30$} & \multicolumn{2}{|c|}{$31-50$} & \multicolumn{2}{|c|}{$\geq \mathbf{5 1}$} & \multicolumn{2}{|c|}{ Overall } \\
\hline & $\%$ & $\mathbf{n}$ & $\%$ & $\mathbf{n}$ & $\%$ & $\mathbf{n}$ & $\%$ & $\mathbf{n}$ \\
\hline$>15$ & 3.47 & 425 & 2.52 & 576 & 1.70 & 329 & 2.68 & 1330 \\
\hline$\geq 16$ & 2.05 & 469 & 1.90 & 1069 & 1.46 & 1713 & 1.73 & 3251 \\
\hline Overall & 2.67 & 894 & 2.14 & 1645 & 1.50 & 2042 & & \\
\hline
\end{tabular}

Number of patients is for 3-year periods (2003-2005).

population were 0.83 for 30 -day mortality and 0.84 for 30 -day operative mortality.

As for 30-day operative mortality, we also presented volume interaction per hospital volume $\times$ surgeon volume (Table 4 ) and conducted subgroup analyses for patient age $(<65$ years and $\geq 65$ years; Table 5) and patient preoperative risk (Table 6). Risk-adjusted mortality rates for each category were calculated by dividing the observed mortality rate by the expected mortality rate at the same hospital and multiplying by the overall $\mathrm{CABG}$ mortality rate of the JACVSD.

\section{Results}

\section{Survey 1 (JATS Database)}

Between January 1, 2001, and December 31, 2004, 82,611 isolated CABG procedures were performed at 540 hospitals in Japan. Average hospital procedural volumes ranged from 0.25 to 292.75 isolated CABG procedures (median, 28 ; interquartile range, 15-49). In Japan, 30 (5.6\%) centers performed 100 or more procedures per year and $133(24.6 \%)$ centers performed fewer than 15 procedures per year. Table 1 displays hospital outcomes and characteristics in the JATS database. High-volume hospitals (those performing $>50$ procedures per year $[\mathrm{n}=131]$ ) had significantly lower mortality rates than those in the other 3 categories. Low-volume hospitals (those performing $\leq 15$ procedures per year $[\mathrm{n}=$ 133]) were more likely to operate in elective cases. As for the JATS database, we also showed unadjusted 30-day mortality rates by procedural volume at 10 intervals (Figure 1). The mortality rate in hospitals performing 41 to $50 \mathrm{CABG}$ procedures per year was $1.91 \%$, and all mortality rates of the following categories (51-60, 61-70, 71-80, 81-90, 91100 , and $\geq 101$ procedures/year) were lower than $2.0 \%$.

\section{Survey 2 (JACVSD)}

Table 2 displays the patient characteristics and outcomes of the JACVSD as a function of hospital volume. Between 2003 and 2005, 4581 isolated CABG operations were performed at 36 participating hospitals. Thirteen of the JACVSD participating hospitals, involving 894 patients, were categorized as medium- to low-volume hospitals (16-30 procedures per year); 14 hospitals, with 1645 CABG patients, were categorized as medium- to high-volume hospitals (31-50 procedures per year); and 9 hospitals, with 2042 CABG patients, were categorized as high-volume $(>50$ procedures per year) hospitals. The medium- to low-volume hospitals were more likely than high-volume centers to operate on patients with chronic lung disease, on patients with hypertension or congestive heart failure, and in emergency or salvage cases. On the basis of preoperative risk factors, the average expected surgical mortality risk rates were $2.4 \%$ in mediumto low-volume hospitals, $2.0 \%$ in medium- to high-volume hospitals, and $1.7 \%$ in high-volume hospitals. Overall, there were 86 cases of 30-day mortality and 117 of 30-day operative mortality (31 patients died in the hospital at $>30$ days). These outcome rates declined in high-volume hospitals in comparison with middle- to low-volume hospitals.

Table 3 displays the effect of volume index on each outcome. Only hospital procedural volume affected 30-day mortality and operative mortality significantly. Table 4 demonstrates the effect of hospital and surgeon procedural

TABLE 5. Unadjusted and risk-adjusted mortality by patient age group $(n=4581)$

\begin{tabular}{|c|c|c|c|c|c|c|}
\hline & \multicolumn{3}{|c|}{ Age $<65$ years } & \multicolumn{3}{|c|}{ Age $\geq 65$ years } \\
\hline & \multicolumn{3}{|c|}{ Hospital CABG volume } & \multicolumn{3}{|c|}{ Hospital CABG volume } \\
\hline & $16-30$ & $31-50$ & $\geq \mathbf{5 0}$ & $16-30$ & $31-50$ & $\geq \mathbf{5 0}$ \\
\hline No. of patients & 287 & 559 & 725 & 607 & 1086 & 1317 \\
\hline Unadjusted mortality & 2.79 & 1.61 & 1.24 & 4.78 & 3.50 & 1.82 \\
\hline \multirow{2}{*}{$\begin{array}{l}\text { Risk-adjusted mortality } \\
P \text { value (hospital volume) }\end{array}$} & 1.53 & 1.23 & 1.03 & 3.28 & 2.62 & 1.73 \\
\hline & & $<.05$ & & & $<.01$ & \\
\hline
\end{tabular}

Number of patients is for 3-year periods (2003-2005). 
TABLE 6. Association between hospital CABG volume and mortality by expected risk $(n=4581)$

\begin{tabular}{|c|c|c|c|c|c|c|}
\hline & \multicolumn{3}{|c|}{ Expected risk $<1.5 \%$} & \multicolumn{3}{|c|}{ Expected risk $>1.5 \%$} \\
\hline & \multicolumn{3}{|c|}{ Hospital CABG volume } & \multicolumn{3}{|c|}{ Hospital CABG volume } \\
\hline & $16-30$ & $31-50$ & $\geq \mathbf{5 0}$ & $16-30$ & $31-50$ & $\geq \mathbf{5 0}$ \\
\hline No. of patients & 432 & 921 & 1252 & 462 & 724 & 790 \\
\hline Unadjusted mortality & 0.69 & 0.98 & 0.16 & 7.36 & 5.25 & 3.92 \\
\hline Risk-adjusted mortality & 0.37 & 0.55 & 0.09 & 5.22 & 3.81 & 3.25 \\
\hline$P$ value (hospital volume) & & NA & & & $<.01$ & \\
\hline
\end{tabular}

Preoperative risk was calculated based on JACVSD 30-day mortality risk model. Results were further adjusted with risk group to ensure constant risk profiles.

Number of patients is for 3-year periods (2003-2005).

volume on risk-adjusted 30 -day operative mortality rates. As there was colinearity between these factors $(r=0.385)$, only hospital procedural volume had a significant effect on 30-day operative mortality $(P<.001)$. Overall, the highest mortality rates $(3.47 \%)$ were observed when patients were treated by low-volume surgeons at middle- to low-volume hospitals, and the best results $(1.46 \%)$ were obtained by high-volume surgeons at high-volume hospitals. We have shown the effect of volume on outcome in patient subgroups (Tables 5 and 6). Regarding the patient age group, the effect of hospital volume was apparent in both groups (age $<65$ years, $P<.05$; age $\geq 65$ years, $P<.01$ ). Patients at expected high operative risk $(>1.5 \%, P<.01)$ demonstrated consistently lower mortality when treated at higher-volume centers. In contrast, among those with a risk of less than $1.5 \%$, there was not a significant volume effect on 30-day operative mortality rates.

\section{Discussion}

Both JATS and JACVSD analyses demonstrated an association between hospital CABG procedural volume and CABG outcome. Although high-quality evaluations of CABG volume-outcome relationships using data from the STS National Cardiac Database ${ }^{27}$ also found an association between volume and outcome, the effects of hospital volume were modest compared with our findings. This may be partly due to the straightforward case mix across volume category (Table 2) or the different distribution of hospital CABG pro- cedural volume in each country. As 4 fairly equal-sized hospital volume break points were 150 or less, 150 to 300,300 to 450, and greater than 450 in the United States, $98.3 \%$ of Japanese centers would be categorized as low-volume hospitals by the US definition. In Japan, there are no standards for opening a cardiac surgery program; they simply proliferate without any regulatory oversight. Although low-volume cardiac surgery programs are loss-making of themselves, many hospitals want a cardiac surgery program not only for the backup of percutaneous coronary intervention but also for prestige as a general hospital. Our results suggest that minimal volume standards of cardiac surgery would be an effective way to improve $\mathrm{CABG}$ outcomes.

The outcome improvement shown in Figure 1 also suggested that the effect of the hospital volume learning curve might be stronger in lower volume distribution in Japan. The reason for the difference regarding volume effect between Japan and other countries may be multifactorial. As many surgeons belong to a single hospital in Japan, information and experiences of conferences on each patient are shared with many cardiac surgeons and other medical staffs in the hospital. Usually, cardiac surgery is performed not by a single consultant but by two or more consultant surgeons with trainees. Characteristics of the Japanese health insurance system might be another reason, because insurance covers a large part of the medical expense with a small individual payment at each operation. ${ }^{28}$ Patients can stay in the hospital

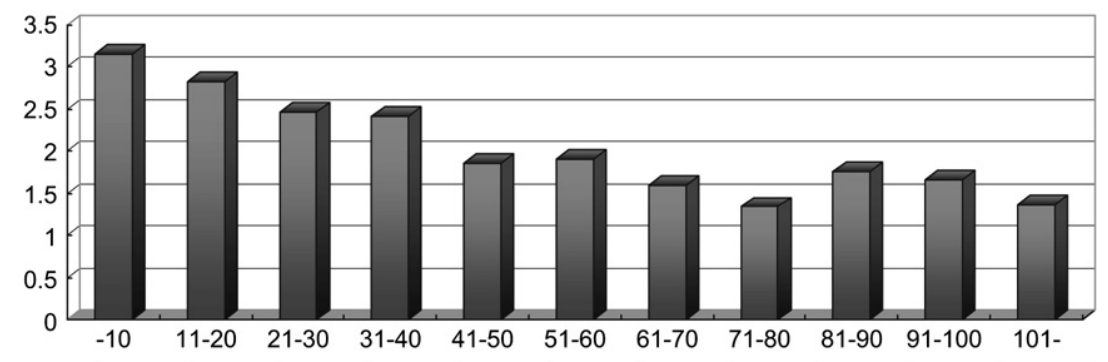

\begin{tabular}{|l|c|c|c|c|c|c|c|c|c|c|c|}
\hline No. of patients & 1780 & 6530 & 9419 & 9479 & 9858 & 6227 & 7494 & 6267 & 3411 & 4931 & 17215 \\
\hline No. of hospitals & 87 & 105 & 94 & 68 & 55 & 28 & 29 & 21 & 10 & 13 & 30 \\
\hline
\end{tabular}

Figure 1. Unadjusted 30-day mortality rates by CABG procedural volume in JATS database (2001-2004). 
beyond 1 or 2 weeks if needed without extra huge expenses, and they can choose any surgeon and hospital they prefer, regardless of their insurance. Surgeons can conduct surgical and medical treatments at the maximum level with less pressure from the hospital and insurance company compared with surgeons in other countries. Moreover, postoperative care in the intensive or critical care unit is maintained mainly by physicians and not by nursing staffs in Japan, which may lead to better care than otherwise. ${ }^{29}$ Because of these differences, minimal volume standards for CABG surgery in Japan might be preferable at lower levels than those in the United States.

As noted in previous studies by Peterson, ${ }^{27}$ Hannan, ${ }^{30}$ and their associates, Table 4 shows that even for very low numbers of cases, low-volume surgeons have substantially better results when they operate at higher-volume hospitals. High-volume hospitals might also be important as teaching institutes. Tables 5 and 6 show that the volume-outcome relationship may be most evident for higher risk patients (older, more comorbidities). In addition to regionalization of cardiac surgery, patient transfer system (eg, transferring high-risk patients to highvolume hospitals quickly) also needs to be developed for better quality of cardiac surgery in Japan.

As for public reporting, hospital-based evaluation might be more relevant than surgeon-based evaluation. Hospital volume index (total adult cardiac procedure volume, hospital CABG-related surgery procedural volume, and hospital CABG-only procedural volume) was significantly associated with 30-day mortality and operative mortality. On the other hand, the surgeon-volume index was not significantly associated with these outcomes. Inasmuch as there are few open-bed hospitals and most surgeons and their teams belong to a single hospital in Japan, a large proportion of surgeon volume might be accounted for by hospital volume. Other studies suggest that individual report cards might discourage surgeons from operating on high-risk patients, because it is surgeons, not hospitals, that choose whether or not to accept a patient for surgery. ${ }^{1,2}$ As for the public reporting regarding outcomes of cardiac surgery in Japan, releasing a hospital-based outcome might be more preferable.

In 2002, the Japanese Ministry of Health, Labor and Welfare set minimal standards by references to hospital procedure volume for medical treatment fees on surgery. ${ }^{31}$ As for cardiac surgery, medical institutes in which the annual cardiac surgery procedural volume was less than 100 had their medical treatment fees lowered by $30 \%$. As many stakeholders objected, these standards were suspended in 2006. Minimal volume standards for CABG surgery in Japan may also be modest not only because most medical institutes (over $60 \%$ ) had been lowered by those standards ${ }^{32}$ but also because of the limitation of procedural volume as a marker of CABG quality. Even when a significant association exists, hospital volume is not a complete predictor of outcome for individual hospitals. Inasmuch as hospital procedural volume embraces physicians' skills, experienced interdisciplinary teams, well-organized care processes, and hospital facilities, it is a necessary factor when outcomes are considered. However, many other parameters (namely, outcome monitoring, compliance with process measures, appropriateness of patient selection for surgery) may also be associated with better outcomes. ${ }^{1,33}$ Thus volume alone is not sufficient for predicting outcome in Japan. In addition, there was wide variance in the results observed among individual centers, particularly those in the low-volume category (Table 1), indicating that not all high-volume providers have better outcomes and not all low-volume providers have worse outcomes. Further studies should include an examination of those parameters to improve the outcomes of individual centers.

Outcome-based evaluation is also an important way to improve quality of CABG surgery. However, surgical mortality has several limitations as an indicator of hospital quality under the present circumstances in Japan because small sample size and low event rates combine to diminish statistical power. ${ }^{34}$ Although volume is not a complete indicator of quality, high-volume providers have, on the whole, better outcomes than low-volume providers. In addition, the effect of hospital procedural volume was significantly associated with better outcomes in almost all patient subgroups (except for low-risk surgery). Regionalization of medical centers on the basis of hospital procedural volume might be effective to improve quality to some extent. However, regionalization has an impact not only on hospital quality, but also on patients' access, staffing of medical professionals, cooperation with other departments in the hospital, and health care expenditure. As for specific health policy recommendations, further analysis is needed to consider these factors. When case loads become large enough to support outcome measurement through regionalization, it is also feasible to base quality assessments on both outcome data and volume (or on one of these).

Several limitations should be noted. In the JACVSD analysis, we excluded centers that submitted fewer than JATS results, because the appropriateness of patient selection for procedural conditions seemed to be important for a volumeoutcome study. A former study also found that high-volume surgeons performed a higher proportion of operations for which the indications were inappropriate than low-volume surgeons. ${ }^{35}$ It is probably appropriate for fair comparison to exclude centers whose reporting is incomplete. Moreover, improving quality of the database is a continuing issue in JACVSD. As for data accuracy, not only data auditing but also to educate each site's input data in correct definition is important.

We thank all members of the Japanese Association for Thoracic Surgery, all members of Japanese Cardiovascular Surgery Database, Takahiro Kiuchi, and Yasuki Kobayashi for their tireless efforts to ensure the timeliness, completeness, and accuracy of the registry data. 


\section{References}

1. Shahian DM. Improving cardiac surgery quality-volume, outcome, process? JAMA. 2004;291:246-8.

2. Burack JH, Impellizzeri P, Homel P. Public reporting of surgical mortality. Ann Thorac Surg. 1999;68:1195-200.

3. Luft HS. The relation between surgical volume and mortality: an exploration of causal factors and alternative models. Med Care. 1980;18: 940-59.

4. Luft HS, Bunker JP, Enthoven AC. Should operations be regionalized? The empirical relation between surgical volume and mortality. $N$ Engl J Med. 1979;301:1364-9.

5. Halm EA, Lee C, Chassin MR. Is volume related to outcome in health care? A systematic review and methodologic critique of the literature. Ann Intern Med. 2002;137:511-20.

6. Hewitt M. Interpreting the volume-outcome relationship in the context of health care quality: workshop summary Institute of Medicine. Washington [DC]: National Academy Press; 2000.

7. Abe H, Tsukada K, Takada T, Ngakawa T. The selection of pancreatic reconstruction techniques gives rise to higher incidences of morbidity: results of the 30th Japan Pancreatic Surgery Questionnaire Survey on pancreatoduodenectomy in Japan. J Hepatobiliary Pancreat Surg. 2005; 12:109-15.

8. Ioka A, Tsukuma H, Ajiki W, Oshima A. Influence of hospital procedure volume on ovarian cancer survival in Japan, a country with low incidence of ovarian cancer. Cancer Sci. 2004;95:233-7.

9. Ioka A, Tsukuma H, Ajiki W, Oshima A. Influence of hospital procedure volume on uterine cancer survival in Osaka. Japan. Cancer Sci. 2005; 96:689-94.

10. Irita K, Kawashima Y, Tsuzaki K, Iwao Y, Seo N, Morita K, et al. [Surgical volume and mortality due to intraoperative critical incidents at Japanese Society of Anesthesiologists certified training hospitals: an analysis of the annual survey in 2002.] Masui. 53:1421-1428. In Japanese.

11. Kinjo K, Sato H, Nakatani D, Mizuno H, Shimizu M, Hishida E, et al. Predictors of length of hospital stay after acute myocardial infraction in Japan. Circ J. 2004;68:809-15.

12. Mitsuyasu S, Hagihara A, Horiguchi H, Nobutomo K. Relationship between total arthroplasty case volume and patient outcome in an acute care payment system in Japan. J Arthroplasty. 2006;21:656-63.

13. Nabae K, Hayashi K, Shirokawa M, Hasegawa T, Hasegawa T. The effect of procedural volume toward digestive cancer. Byouin Kanri. 2003;40:39-51.

14. Nomura E, Tsukuma H, Ajiki T, Oshima A. Population-based study of relationship between hospital surgical volume and 5-year survival of stomach cancer patients in Osaka Japan. Cancer Sci. 2003;94:998-1002.

15. Saika K, Ohno Y, Tanaka H, Hasegawa T, Tsukuma H, Oshima A. The trend of the effect of surgical volume up to 5 years after resection for stomach and lung cancer patients. IT Healthcare. 2007;1,2:42-9.

16. Tsuchihashi M, Tsutsui H, Tada H, Shihara M, Takeshita A, Kono S. Volume-outcome relation for hospitals performing angioplasty for acute myocardial infarction-results from the nationwide Japanese registry. Circ J. 2004;68:887-91.

17. Haga Y, Ikei S, Wada Y, Takeuchi H, Sameshima H, Kimura O, et al. Evaluation of an estimation of physiologic ability and surgical stress (E-PASS) scoring system to predict postoperative risk: a multicenter prospective study. Surg Today. 2001;31:569-74.

18. Fujino Y, Suzuki Y, Ajiki T, Tanioka Y, Ku Y, Kuroda Y. Risk factor influencing pancreatic leakage and the mortality after pancreaticoduode- nectomy in a medium-volume hospital. Hepatogastroenterology. 2002; 49:1124-9.

19. Fujita T, Yamazaki Y. influence of surgeon's volume on early outcome after total Gastrectomy. Eur J Surg. 2002;168:535-8.

20. Yada I, Wada H, Fujita H. Thoracic and cardiovascular surgery in Japan during 2002: annual report by the Japanese Association for Thoracic Surgery. Jpn J Thorac Cardiovasc Surg. 2004;52:491-508.

21. Yada I, Wada H, Shinoda M, Yasuda K. Thoracic and cardiovascular surgery in Japan during 2001: annual report by the Japanese Association for Thoracic Surgery. Jpn J Thorac Cardiovasc Surg. 2003;51:699-716.

22. Kazui T, Osada H, Fujita H. Thoracic and cardiovascular surgery in Japan during 2004. Jpn J Thorac Cardiovasc Surg. 2006;54:363-85.

23. Kazui T, Wada H, Fujita H. Thoracic and cardiovascular surgery in Japan during 2003: annual report by The Japanese Association for Thoracic Surgery. Jpn J Thorac Cardiovasc Surg. 2005;53:517-36.

24. Edmunds LHJ, Clark RE, Cohn LH, Grunkemeler GL, Miller DC, Weisel RD. Guidelines for reporting morbidity and mortality after cardiac valvular operations; the American Association for Thoracic Surgery, Ad Hoc Liaison Committee for Standardizing Definitions of Prosthetic Heart Valve Morbidity. Ann Thorac Surg. 1996;62:932-5.

25. Shroyer AL, Coombs LP, Peterson ED, Eiken MC, DeLong ER, Chen A, et al. The Society of Thoracic Surgeons: 30-day operative mortality and morbidity risk models. Ann Thorac Surg. 2003;75:1856-64; discussion 1864-5.

26. Berlin JA, Kimmel SE, Ten Have TR, Sammel MD. An empirical comparison of several clustered data approaches under confounding due to cluster effects in the analysis of coronary angiography. Biometrics. 1999;55:470-6.

27. Peterson ED, Coombs LP, DeLong ER, Haan CK, Ferguson TB. Procedural volume as a marker of quality for CABG surgery. JAMA. 2004; 291:195-201.

28. Matsuda S, Yamamoto M. Long-term care insurance and integrated care for the aged in Japan. Int J Integr Care. 2001;1:e28.

29. Yoshiya I, Baik SW. Critical care in Japan and Korea. The market of excellence. Crit Care Clin. 1997;13:267-85.

30. Hannan EL, Wu C, Ryan TJ, Bennett E, Culliford AT, Jefferey PG, et al. Do hospitals and surgeons with higher coronary artery bypass graft surgery volumes still have lower risk-adjusted mortality rates? Circulation. 2003; 108:795-801.

31. Miyata H, Motomura N, Takamoto S. Regionalization of cardiac surgery in Japan I. Effect of procedural volume on outcome of CABG surgery. Kyobu Geka. 2007a;60:334-43. In Japanese.

32. Miyata H, Kondo JM, Motomura N, Fushimi K, Takamoto S. Regionalization of cardiac surgery in Japan II. Estimating the multiple effect of regionalization in cardiac surgery. Kyobu Geka. 2007;60:747-53. In Japanese.

33. Ferguson BT, Peterson ED, Coombs LP, Eiken MC, Carey ML, Grover FL, et al. Use of continuous quality improvement to increase use of process measures in patients undergoing coronary artery bypass graft surgery. JAMA. 2003;290:49-56.

34. Dimick JB, Welch HG, Birkmeyer JD. Surgical mortality as an indicator of hospital quality: the problem with small sample size. JAMA. 2004, 292:847-51.

35. Brook RH, Park RE, Chassin MR, Kosecoff J, Keesey J, Solomon DH. Carotid endarterectomy for elderly patients: predicting complications. Ann Intern Med. 1990;113:747-53. 